\title{
A Class of Iterative Methods for Solving Nonlinear Equations with Optimal Fourth-order Convergence
}

\author{
J. P. Jaiswal \\ Department of Mathematics, Maulana Azad National Institute of Technology, Bhopal, M.P., 462051, India \\ *Corresponding Author: jaiprakashjaiswal@manit.ac.in
}

Copyright (c)2014 Horizon Research Publishing All rights reserved.

\begin{abstract}
In this paper we construct a new third-order iterative method for solving nonlinear equations for simple roots by using inverse function theorem. After that a class of optimal fourth-order methods by using one function and two first derivative evaluations per full cycle is given which is obtained by improving the existing third-order method with help of weight function. Some physical examples are given to illustrate the efficiency and performance of our methods.
\end{abstract}

Keywords Newton Method, Order of Convergence, Optimal Order, Inverse Function, Weight Function

Mathematics Subject Classification (2000). 41A25, 65D99

\section{Introduction}

Nonlinear equations plays an important role in science and engineering. Finding an analytic solution is not always possible. Therefore, numerical methods are used in such situations. The classical Newton's method is the best known iterative method for solving nonlinear equations. To improve the local order of convergence and efficiency index, many modified third-order methods have been presented in literature. For detail we refer [[16], [9], [1], [15], [5]] and references therein.

Recently Ardelean [3] established Bisectrix Newton's Method (BN), which is given by

$$
x_{n+1}=x_{n}-\frac{\left(f^{\prime}\left(x_{n}\right)+f^{\prime}\left(y_{n}\right)\right) f\left(x_{n}\right)}{f^{\prime}\left(x_{n}\right) f^{\prime}\left(y_{n}\right)+\sqrt{\left(1+f^{\prime}\left(x_{n}\right)^{2}\right)\left(1+f^{\prime}\left(y_{n}\right)^{2}\right)}-1}, n \geq 0,
$$

where $y_{n}=x_{n}-\frac{f\left(x_{n}\right)}{f^{\prime}\left(x_{n}\right)}$. The method is third-order convergent for simple roots and its efficiency index is $3^{1 / 3}=1.4422$.

Weerakoon et al. [16] used Newton's theorem

$$
f(x)=f\left(x_{n}\right)+\int_{x_{n}}^{x} f^{\prime}(t) d t
$$

and approximated the integral by trapezoidal rule, i.e.

$$
\int_{x_{n}}^{x} f^{\prime}(t) d t=\frac{\left(x-x_{n}\right)}{2}\left[f^{\prime}\left(x_{n}\right)+f^{\prime}(x)\right]
$$

obtained the following variant of the Newton method

$$
x_{n+1}=x_{n}-\frac{2 f\left(x_{n}\right)}{f^{\prime}\left(x_{n}\right)+f^{\prime}\left(y_{n}\right)},
$$

where $y_{n}=x_{n}-\frac{f\left(x_{n}\right)}{f^{\prime}\left(x_{n}\right)}$. It is shown that this is third-order. Many authors used this idea to approximate the integral $\int_{x_{n}}^{x} f^{\prime}(t) d t$ by different rule. For more detail, one can see [ [12], [13], [14], [8], [9], [15], [5] ] and the references there in.

If we approximated the integral in (1.2) by

$$
\int_{x_{n}}^{x} f^{\prime}(t) d t=\left(x-x_{n}\right)\left[\frac{f^{\prime}\left(x_{n}\right) f^{\prime}(x)+\sqrt{\left(1+f^{\prime}\left(x_{n}\right)^{2}\right)\left(1+f^{\prime}(x)^{2}\right)}-1}{\left(f^{\prime}\left(x_{n}\right)+f^{\prime}(x)\right)}\right],
$$


we get the same formula $(1.1)$.

Next Homeier [9] used Newton's theorem (1.2) for the inverse function $x=f^{-1}(y)=g(y)$ instead of $y=f(x)$, that is

$$
g(y)=g\left(y_{n}\right)+\int_{y_{n}}^{y} g^{\prime}(s) d s .
$$

Then the method (1.4) takes the form

$$
x_{n+1}=x_{n}-\frac{f\left(x_{n}\right)}{2}\left[\frac{1}{f^{\prime}\left(x_{n}\right)}+\frac{1}{f^{\prime}\left(y_{n}\right)}\right],
$$

where $y_{n}=x_{n}-\frac{f\left(x_{n}\right)}{f^{\prime}\left(x_{n}\right)}$. This method is again third-order.

Here we state following definitions:

Definition 1.1. Let $f(x)$ be a real function with a simple root $\alpha$ and let $x_{n}$ be a sequence of real numbers that converge towards $\alpha$. The order of convergence $m$ is given by

$$
\lim _{n \rightarrow \infty} \frac{x_{n+1}-\alpha}{\left(x_{n}-\alpha\right)^{m}}=\zeta \neq 0
$$

where $\zeta$ is the asymptotic error constant and $m \in R^{+}$.

Definition 1.2. Let $\beta$ be the number of function evaluations of the new method. The efficiency of the new method is measured by the concept of efficiency index $[17,11]$ and defined as

$$
\mu^{1 / \beta}
$$

where $\mu$ is the order of the method.

Kung and Traub [10] presented a hypothesis on the optimality of roots by giving $2^{n-1}$ as the optimal order. This means that the Newton iteration by two evaluations per iterations is optimal with 1.414 as the efficiency index. By taking into account the optimality concept many authors have tried to build iterative methods of optimal higher order of convergence.

This paper is organized as follows: in section 2, we describe the new third-order iterative method by using the concept of inverse function theorem. In the next section we optimize the method of Chun et. al [4] with help of weight function. Finally in the last section we give some physical example and our new methods are compared in the performance with some well known methods.

\section{Development of the method and convergence analysis}

In this section we use the concept of inverse function to derive variants of Bisectrix Newton's Method. In the formula (1.1), function $y=f(x)$ has been used. Here we use inverse function $x=f^{-1}(y)=g(y)$ instead of $y=f(x)$. Then we have

$$
\begin{aligned}
g(y) & =g\left(y_{n}\right)+\int_{y_{n}}^{y} g^{\prime}(s) d s \\
& =g\left(y_{n}\right)+\left(y-y_{n}\right)\left[\frac{g^{\prime}\left(y_{n}\right) g^{\prime}(y)+\sqrt{\left(1+g^{\prime}\left(y_{n}\right)^{2}\right)\left(1+g^{\prime}(y)^{2}\right)}-1}{\left(g^{\prime}\left(y_{n}\right)+g^{\prime}(y)\right)}\right],
\end{aligned}
$$

where $y_{n}=f\left(x_{n}\right)$. Now using the fact that $g^{\prime}(y)=\left(f^{-1}\right)^{\prime}(y)=\left[f^{\prime}(x)\right]^{-1}$ and that $y=f(x)=0$, we obtain the following method:

$$
x_{n+1}=x_{n}-f\left(x_{n}\right)\left[\frac{1+\sqrt{\left(1+f^{\prime}\left(x_{n}\right)^{2}\right)\left(1+f^{\prime}\left(y_{n}\right)^{2}\right)}-f^{\prime}\left(x_{n}\right) f^{\prime}\left(y_{n}\right)}{f^{\prime}\left(x_{n}\right)+f^{\prime}\left(y_{n}\right)}\right] \text {. }
$$

where

$$
y_{n}=x_{n}-\frac{f\left(x_{n}\right)}{f^{\prime}\left(x_{n}\right)} .
$$

Now we prove that order of convergence of this method is also three.

Theorem 2.1. Let the function $f$ have sufficient number of continuous derivatives in a neighborhood of $\alpha$ which is a simple root off, then the method (2.2) has third-order convergence. 
Proof. Let $e_{n}=x_{n}-\alpha$ be the error in the $n^{t h}$ iterate and $c_{h}=\frac{f^{(h)}(\alpha)}{h !}, h=1,2,3 \ldots$ We provide the Taylor series expansion of each term involved in (2.2). By Taylor expansion around the simple root in the $n^{\text {th }}$ iteration, we have

$$
f\left(x_{n}\right)=f^{\prime}(\alpha)\left[e_{n}+c_{2} e_{n}^{2}+c_{3} e_{n}^{3}+c_{4} e_{n}^{4}+c_{5}^{5} e_{n}^{5}+c_{6} e_{n}^{6}+O\left(e_{n}^{7}\right)\right]
$$

and, we have

$$
f^{\prime}\left(x_{n}\right)=f^{\prime}(\alpha)\left[1+2 c_{2} e_{n}+3 c_{3} e_{n}^{2}+4 c_{4} e_{n}^{3}+5 c_{5}^{5} e_{n}^{4}+6 c_{6} e_{n}^{5}+O\left(e_{n}^{6}\right)\right]
$$

Further more it can be easily find

$$
\frac{f\left(x_{n}\right.}{f^{\prime}\left(x_{n}\right)}=e_{n}-c_{2} e_{n}^{2}+\left(2 c_{2}^{2}-2 c_{3}\right) e_{n}^{3}+\ldots \ldots . .+O\left(e_{n}^{6}\right) .
$$

By considering this relation, we obtain

$$
y_{n}=\alpha+c_{2} e_{n}^{2}+2\left(c_{3}-c_{2}^{2}\right) e_{n}^{3}+\ldots \ldots+O\left(e_{n}^{6}\right) .
$$

At this time, we should expand $f^{\prime}\left(y_{n}\right)$ around the root by taking into consideration (2.7). Accordingly, we have

$$
f^{\prime}\left(y_{n}\right)=f^{\prime}(\alpha)\left[1+2 c_{2}^{2} e_{n}^{2}+\left(4 c_{2} c_{3}-4 c_{2}^{3}\right) e_{n}^{3}+\ldots+O\left(e_{n}^{6}\right)\right] .
$$

By consider the above mentioned relations $(2.4),(2.5)$ and $(2.8)$ in the equation $(2.2)$, we can find

$$
e_{n+1}=\left(\frac{c_{2}^{2}}{1+f^{\prime}(\alpha)^{2}}+\frac{c_{3}}{2}\right) e_{n}^{3}+O\left(e_{n}^{4}\right) .
$$

This confirms the result.

\section{Optimal fourth-order iterative method}

By using circle of curvature concept Chun et. al. [4] constructed a third-order iterative methods defined by

$$
\begin{aligned}
y_{n} & =x_{n}-\frac{f\left(x_{n}\right)}{f^{\prime}\left(x_{n}\right)}, \\
x_{n+1} & =x_{n}-\frac{1}{2}\left[3-\frac{f^{\prime}\left(y_{n}\right)}{f^{\prime}\left(x_{n}\right)}\right] \frac{f\left(x_{n}\right)}{f^{\prime}\left(x_{n}\right)} .
\end{aligned}
$$

The order of this method three is with three (one derivative and two function) evaluations per full iteration. Clearly its efficiency index $\left(3^{1 / 3} \approx 1.4422\right)$ is not high (optimal $=\left(4^{1 / 3} \approx 1.5844\right)$. We now make use of weight function approach to build our optimal class based on (3.1) by a simple change in its first step. Thus we consider

$$
\begin{aligned}
y_{n} & =x_{n}-a \frac{f\left(x_{n}\right)}{f^{\prime}\left(x_{n}\right)}, \\
x_{n+1} & =x_{n}-\frac{1}{2}\left[3-\frac{f^{\prime}\left(y_{n}\right)}{f^{\prime}\left(x_{n}\right)}\right] \frac{f\left(x_{n}\right)}{f^{\prime}\left(x_{n}\right)} \times G(t) .
\end{aligned}
$$

where $G(t)$ is a real-valued weight function with $t=\frac{f^{\prime}\left(y_{n}\right)}{f^{\prime}\left(x_{n}\right)}$ and $a$ is a real constant. The weight function should be chosen such that order of convergence arrives at optimal level four without using more function evaluations. The following theorem indicates under what conditions on the weight functions and constant $a$ in (3.2), the order of convergence will arrive at the optimal level four:

Theorem 3.1. Let the function $f$ have sufficient number of continuous derivatives in a neighborhood of $\alpha$ which is a simple root of $f$, then the method (3.2) has fourth-order convergence, when $a=2 / 3$ and the weight function $G(t)$ satisfies the following conditions

$$
G(1)=1, G^{\prime}(1)=\frac{-1}{4}, G^{\prime \prime}(1)=2,\left|G^{(3)}(1)\right| \leq+\infty
$$

and the error equation is given by (3.8).

Proof. Using (2.4) and (2.5) and $a=2 / 3$ in the first step of (3.2), we have

$$
y_{n}=\alpha+\frac{e_{n}}{3}+\frac{2 c_{2} e_{n}^{2}}{3}+\frac{4\left(c_{3}-c_{2}^{2}\right) e_{n}^{3}}{3}+\ldots+O\left(e_{n}^{6}\right) .
$$


Now we should expand $f^{\prime}\left(y_{n}\right)$ around the root by taking into consideration (3.4). Thus, we have

$$
f^{\prime}\left(y_{n}\right)=f^{\prime}(\alpha)\left[1+\frac{2 c_{2} e_{n}}{3}+\frac{\left(4 c_{2}^{2}+c_{3}\right) e_{n}^{2}}{3}+\ldots+O\left(e_{n}^{6}\right)\right] .
$$

Furthermore, we have

$$
\frac{f^{\prime}\left(y_{n}\right)}{f^{\prime}\left(x_{n}\right)}=1-\frac{2 c_{2}}{3} e_{n}+\left(4 c_{2}^{2}-\frac{8 c_{3}}{3}\right) e_{n}^{2}+\ldots+O\left(e_{n}^{6}\right)
$$

By virtue of (3.6) and (3.3), we attain

$$
\begin{aligned}
& \frac{1}{2}\left[3-\frac{f^{\prime}\left(y_{n}\right)}{f^{\prime}\left(x_{n}\right)}\right] \frac{f\left(x_{n}\right)}{f^{\prime}\left(x_{n}\right)} \times G(t) \\
& =e_{n}+\left[c_{2} c_{3}-\frac{c_{4}}{9}+-\frac{1}{81}\left\{309+32 H^{(3)}(1)\right\} c_{2}^{3}\right] e_{n}^{4}+O\left(e_{n}^{5}\right) .
\end{aligned}
$$

Finally using (3.7) in (3.2), we can have the following general equation, which reveals the fourth-order convergence

$$
\begin{aligned}
& e_{n+1}=x_{n+1}-\alpha \\
& =x_{n}-\frac{1}{2}\left[3-\frac{f^{\prime}\left(y_{n}\right)}{f^{\prime}\left(x_{n}\right)}\right] \frac{f\left(x_{n}\right)}{f^{\prime}\left(x_{n}\right)} \times G(t)-\alpha \\
& =\left[-c_{2} c_{3}+\frac{c_{4}}{9}+\frac{1}{81}\left\{309+32 G^{(3)}(1)\right\} c_{2}^{3}\right] e_{n}^{4}+O\left(e_{n}^{5}\right) .
\end{aligned}
$$

This proves the theorem.

It is obvious that our novel class of iterations requires three evaluations per iteration, i.e. two first derivative and one function evaluations. Thus our new methods are optimal. Now by choosing appropriate weight functions as presented in (3.2), we can give number of optimal two-step iterative methods. Here we are giving one of them as follows:

$$
\begin{aligned}
y_{n} & =x_{n}-\frac{2}{3} \frac{f\left(x_{n}\right)}{f^{\prime}\left(x_{n}\right)}, \\
x_{n+1} & =x_{n}-\frac{1}{2}\left[3-\frac{f^{\prime}\left(y_{n}\right)}{f^{\prime}\left(x_{n}\right)}\right]\left[\frac{9}{4}-\frac{9}{4} \frac{f^{\prime}\left(y_{n}\right)}{f^{\prime}\left(x_{n}\right)}+\left(\frac{f^{\prime}\left(y_{n}\right)}{f^{\prime}\left(x_{n}\right)}\right)^{2}\right] \frac{f\left(x_{n}\right)}{f^{\prime}\left(x_{n}\right)} .
\end{aligned}
$$

where its error equation is

$$
e_{n+1}=\left[-c_{2} c_{3}+\frac{c_{4}}{9}+\frac{309}{81} c_{2}^{3}\right] e_{n}^{4}+O\left(e_{n}^{5}\right)
$$

\section{Examples}

In this section we give some physical examples and compare our methods with other some well known methods. Here all the computations have been done by using Mathematica 8 . We consider the number of decimal places as follows: 200 digits floating point $($ SetAccuracy $=200)$ with SetAccuracy command. The test of examples from $(4.1)$ to $(4.3)$ are listed in the Table 1 to 3 respectively. We compare the performance of our third-order method (M1) (2.2) and fouth-order (M2) (3.10) method with Newton method (NM), Weerakkon method (WKM) (1.4), Hommeier method (HMM) (1.7), Bisectrix Newton method (BNM) (1.1), Chun method (CHM) (3.1), method (6) (KHM) of [18] and method (17) (SNM) of [7] respectively.

Example 4.1[2] Consider Plank's radiation law

$$
\phi(\lambda)=\frac{8 \pi c h \lambda^{-5}}{e^{c h / \lambda k T}-1}
$$

where $\lambda$ is the wavelength of the radiation, $t$ is the absolute temperature of the blackbody, $k$ is Boltzmann's constant, $h$ is the Planck's constant and $c$ is the speed of light. This formula calculate the energy density within an isothermal blackbody. Now we want to find wavelength $\lambda$ which maximize energy density $\phi(\lambda)$. For maximum of $\phi(\lambda)$, it can easily seen that

$$
\frac{(c h / \lambda k T) e^{c h / \lambda k T}}{e^{c h / \lambda k T}-1}=5 .
$$

Let $x=c h / \lambda k T$, then it becomes

$$
e^{-x}=1-x / 5
$$


Table 1. Errors Occurring in the estimates of the root of function $f_{1}$ by the methods described below with initial guess $x_{0}=5$.

\begin{tabular}{llll}
\hline Methods & $\left|x_{1}-\alpha\right|$ & $\left|x_{2}-\alpha\right|$ & $\left|x_{3}-\alpha\right|$ \\
\hline NM & $0.21464 \mathrm{e}-4$ & $0.83264 \mathrm{e}-11$ & $0.12530 \mathrm{e}-23$ \\
WKM & $0.11208 \mathrm{e}-6$ & $0.37810 \mathrm{e}-23$ & $0.14517 \mathrm{e}-72$ \\
HMM & $0.12544 \mathrm{e}-6$ & $0.59456 \mathrm{e}-23$ & $0.63310 \mathrm{e}-72$ \\
BNM & $0.11256 \mathrm{e}-6$ & $0.38466 \mathrm{e}-23$ & $0.15352 \mathrm{e}-72$ \\
CHM & $0.98734 \mathrm{e}-7$ & $0.22705 \mathrm{e}-23$ & $0.27611 \mathrm{e}-73$ \\
M1 & $0.11256 \mathrm{e}-6$ & $0.38466 \mathrm{e}-23$ & $0.15352 \mathrm{e}-72$ \\
SNM & $0.14780 \mathrm{e}-5$ & $0.47702 \mathrm{e}-23$ & $0.51761 \mathrm{e}-93$ \\
KHM & $0.47426 \mathrm{e}-9$ & $0.16796 \mathrm{e}-40$ & $0.26148 \mathrm{e}-166$ \\
M2 & $0.42864 \mathrm{e}-9$ & $0.10085 \mathrm{e}-40$ & $0.30899 \mathrm{e}-167$ \\
\hline
\end{tabular}

Now the above equation can be rewritten as

$$
f_{1}(x)=e^{-x}-1+x / 5 \text {. }
$$

Our aim to find the root of the equation $f_{1}(x)=0$. Clearly zero is its one root, which is not of our interest. If we take $x=5$, then R.H.S. of (4.2) becomes zero and L.H.S. is $e^{-5} \approx 6.74 \times 10^{-3}$. This implies one root of the equation $f_{1}(x)=0$ is near to 5. So that here we compare some well known methods to our methods with initial guess 5, which are given in table 1.

Example 4.2 [6] The depth of embedment $x$ of a sheet-pile wall is governed by the equation:

$$
x=\frac{x^{3}+2.87 x^{2}-10.28}{4.62} .
$$

It can be rewritten as

$$
f_{2}(x)=\frac{x^{3}+2.87 x^{2}-10.28}{4.62}-x .
$$

An engineer has estimated the depth to be $x=2.5$. Here we find the root of the equation $f_{2}(x)=0$ with initial guess 2.5 and compare some well known methods to our methods, which are given in table 2.

Table 2. Errors Occurring in the estimates of the root of function $f_{2}$ by the methods described below with initial guess $x_{0}=2.5$.

\begin{tabular}{llll}
\hline Methods & $\left|x_{1}-\alpha\right|$ & $\left|x_{2}-\alpha\right|$ & $\left|x_{3}-\alpha\right|$ \\
\hline NM & $0.85925 \mathrm{e}-1$ & $0.32675 \mathrm{e}-2$ & $0.50032 \mathrm{e}-5$ \\
WKM & $0.18271 \mathrm{e}-1$ & $0.14770 \mathrm{e}-5$ & $0.79610 \mathrm{e}-18$ \\
HMM & $0.49772 \mathrm{e}-2$ & $0.33027 \mathrm{e}-8$ & $0.95318 \mathrm{e}-27$ \\
BNM & $0.54594 \mathrm{e}-2$ & $0.63617 \mathrm{e}-8$ & $0.10016 \mathrm{e}-25$ \\
CHM & $0.27815 \mathrm{e}-1$ & $0.95903 \mathrm{e}-5$ & $0.41254 \mathrm{e}-15$ \\
M1 & $0.54594 \mathrm{e}-2$ & $0.63617 \mathrm{e}-8$ & $0.10016 \mathrm{e}-25$ \\
SNM & $0.26594 \mathrm{e}-1$ & $0.32982 \mathrm{e}-6$ & $0.76311 \mathrm{e}-26$ \\
KHM & $0.14965 \mathrm{e}-1$ & $0.45484 \mathrm{e}-7$ & $0.40826 \mathrm{e}-29$ \\
M2 & $0.80338 \mathrm{e}-2$ & $0.15138 \mathrm{e}-8$ & $0.19455 \mathrm{e}-35$ \\
\hline
\end{tabular}

Example 4.3 [6] The vertical stress $\sigma_{z}$ generated at point in an elastic continuum under the edge of a strip footing supporting a uniform pressure $q$ is given by Boussinesq's formula to be:

$$
\sigma_{z}=\frac{q}{\pi}\{x+\operatorname{Cos} x \operatorname{Sin} x\}
$$

A scientist is interested to estimate the value of $x$ at which the vertical stress $\sigma_{z}$ will be 25 percent of the footing stress $q$. Initially it is estimated that $x=0.4$. The above can be rewritten as for $\sigma_{z}$ is equal to 25 percent of the footing stress $q$ :

$$
f_{3}(x)=\frac{x+\operatorname{Cos} x \operatorname{Sin} x}{\pi}-\frac{1}{4}
$$

Now we find the root of the equation $f_{3}(x)=0$ with initial guess 0.4 and compare some well known methods to our methods, which are given in table 3 . 
Table 3. Errors Occurring in the estimates of the root of function $f_{3}$ by the methods described below with initial guess $x_{0}=0.4$.

\begin{tabular}{llll}
\hline Methods & $\left|x_{1}-\alpha\right|$ & $\left|x_{2}-\alpha\right|$ & $\left|x_{3}-\alpha\right|$ \\
\hline NM & $0.10737 \mathrm{e}-3$ & $0.50901 \mathrm{e}-8$ & $0.11442 \mathrm{e}-16$ \\
WKM & $0.20631 \mathrm{e}-6$ & $0.53436 \mathrm{e}-21$ & $0.92858 \mathrm{e}-65$ \\
HMM & $0.52795 \mathrm{e}-6$ & $0.19743 \mathrm{e}-19$ & $0.10325 \mathrm{e}-59$ \\
CHM & $0.93064 \mathrm{e}-6$ & $0.20624 \mathrm{e}-18$ & $0.22446 \mathrm{e}-56$ \\
M1 & $0.42239 \mathrm{e}-7$ & $0.13373 \mathrm{e}-23$ & $0.42435 \mathrm{e}-73$ \\
SNM & $0.86290 \mathrm{e}-7$ & $0.78612 \mathrm{e}-28$ & $0.54150 \mathrm{e}-112$ \\
KHM & $0.52074 \mathrm{e}-7$ & $0.67319 \mathrm{e}-29$ & $0.18803 \mathrm{e}-116$ \\
M2 & $0.25102 \mathrm{e}-8$ & $0.17099 \mathrm{e}-30$ & $0.36814 \mathrm{e}-123$ \\
\hline
\end{tabular}

\section{Conclusion}

In this present paper we have given a new third-order and a class of the optimal fourth-order iterative methods for simple roots for solving nonlinear equations. The third-order method is obtained by using inverse function theorem and the class optimal fourth-order method is obtained with help of weight function using in the existing third-order method without using any function evaluations. Three physical examples are given to illustrate the superior performance of our methods by comparing them with some well existing third and fourth-order iterative methods.

\section{REFERENCES}

[1] A. Y. Ozban: Some new variants of Newton's method, Appl. Math. Letter, 17 (2004), 677-682.

[2] B. Bradie: A Friendly Introduction to Numerical Analysis, Pearson Education Inc, New Delhi, (2006).

[3] C. Ardelean: A new third-order Newton-type iterative method for solving nonlinear equations, Appl. Math. Comput. 219 (2013), 9856-9864.

[4] C. Chun and Y. I. Kim: Several new third-Order iterative methods for solving nonlinear equations, Acta Appl. Math. 109 (2010), 1053-1063.

[5] D. Jain: Families of Newton-like methods with fourth-order convergence, International Journal of Computer Mathematics, 90 (5) (2013), 1072-1082.

[6] D. V. Griffithms and I. M. Smith: Numerical methods for engineers, Second Edition, Chapman and Hall/CRC (Taylor and Francis Grpup), Special Indian Edition (2011).

[7] F. Soleymani: Two new classes of optimal Jarratt-type fourth order methods, Applied Mathematics Letter, 25 (2012), 847-853.

[8] H. H. H. Homeier: A modified Newton method for root finding with cubic convergence, J. Comput. Appl. Math. 157 (2003), 227-230.

[9] H. H. H. Homeier: On Newton-type methods with cubic convergence, J. Comput. Appl. Math. 176 (2005), $425-432$.

[10] H. T. Kung and J. F. Traub: Optimal order of one-point and multipoint iteration, JCAM 21 (1974), 643-651 .

[11] J. F. Traub: Iterative methods for solution of equations chelsea Publishing, New York, NY, USA (1997).

[12] M. Frontini: Hermite interpolation and a new iterative method for the computation of the roots of non-linear equations, Calcolo 40 (2003), 109-119.

[13] M. Frontini and E. Sormani: Modified Newton's method with third-order convergence and multiple roots, J. Comput. Appl. Math. 156 (2003), 345-354.

[14] M. Frontini and E. Sormani: Some variant of Newton's method with third-order convergence, Appl. Math. Comput. 140 (2003), 419-426.

[15] P. Wang: A third-order family of Newton-like iteration methods for solving nonlinear equations, J. Numer. Math. Stoch. 3 (2011), 13-19.

[16] S. Weerakoon, T. G. I. Fernando: A variant of Newton's method with accelerated third-order convergence, Appl.Math. Lett.13 (2000) 87-93.

[17] W. Gautschi: Numerical Analysis: An Introduction Birkhauser, Barton, Mass, USA (1997). 
[18] S. K. Khattri iand S. Abbasbandy: Optimal fourth order family of iterative methods, Mat. Vesnik 63 (2011) 67-72. 